\title{
Current situation and prospect of individualized oncology medicine in precision medicine
}

\author{
Zhenqing Feng* \\ Key Laboratory of Antibody Technique of Ministry of Health, Nanjing Medical University, Nanjing, Jiangsu, 211166, China
}

Keywords: tumor, pathogenesis, precision medicine, individualized therapy

Malignant tumors have always been harmful to human health and one of the major causes of death worldwide. According to recent data, there were about 14 million new cases of cancer in the world and 8.2 million cases of cancer-related deaths in 2012. Of which, developing countries accounted for about $57 \%$ of new cases of cancer, and up to $65 \%$ of the total global number of deaths. More recent statistics report that as of 2015, there are 4.3 million new cancer cases and 2.8 million deaths in China alone. The digestive system tumors, including gastric cancer, liver cancer, esophageal cancer and colorectal cancer occupy 4 positions among the top 5 tumors of cancer incidence and mortality in China.

As the study of the etiology, pathogenesis and regulation of malignant tumors has continued throughout the century, there have been many hypotheses, from gene mutation, chromosome translocation, epigenetic alterations, stem cell origin, tumor stem cell, tumor recurrence and metastasis, to signal regulation networks of heredity, evolution, development and metabolism. In 2000, Hanahan and Weinberg expounded the six characteristics of tumor cells systematically in the highly regarded "The hallmarks of cancer" published in CELL, which included sell-sufficiency in growth signals, insensitivity to anti-growth signals, evading apoptosis, limitless replicative potential, sustained an-

\footnotetext{
"Correspondence to: Zhenqing Feng, Key Laboratory of Antibody Technique of Ministry of Health, Nanjing Medical University, Nanjing, Jiangsu, 211166, China. Tel:+86-25-86868701, Fax: +86-2586862726, E-mails: fengzhenqing@njmu.edu.cn.
}

giogenesis, tissue invasion and metastasis, emphasizing the central position of tumor cells in tumorigenesis. Ten years later, the concept of "tumor microenvironment" was first proposed in an updated version of the article "The next generation" published in CELL again. The paper pointed out that the "tumor microenvironment" is another significant feature of tumors and added the other four characteristics of tumor cells, including immune destruction avoidance, genome instability and mutation, cellular energetics deregulation and tumorpromoting inflammation. Autophagy is a common and important life phenomenon in eukaryotic cells and is a programmed intracellular degradation mechanism. In recent years, a large number of studies have shown that autophagy is closely related to the occurrence and development of cancers. It has been demonstrated that autophagy has an inhibitory action in the early stages of tumor progression. The 2016 Nobel Prize in Physiology or Medicine was awarded to Japanese scientist Yoshinori Ohsumi who discovered autophagy genes for the first time. His findings confirmed that autophagy was involved in the development of multiple diseases of the human body and provided innovative ideas for our research on tumor pathogenesis and cancer prevention.

With the progress of techniques in biochemistry and molecular biology and the extensive application of large-scale gene sequencing technology, the development and progress of malignant tumors have become better understood and the significant progress has been made in the molecular diagnosis and targeted therapy of some tumors. In January 2015, US President Barack Obama launched the "precision medicine" initiatively in the State of the Union speech. In March of 
the same year, the Ministry of Science and Technology of China held its first academic meeting on precision medicine strategy and put forward the plan of precision medicine of China. In March 2016, the Ministry of Science and Technology of China promulgated the guidelines for key special projects of "precision medical research". The aims are to establish a multi-level precision medical knowledge base system, a safe and operable biomedical big data sharing platform, to break through the clinical application technology of the new generation life omics and big data analysis technology of biomedicine, and to establish innovative large-scale research and development system of biomarkers, targets and formulations for disease early warning, diagnosis, treatment and efficacy evaluation.

Genome wide association studies (GWAS) as a genetic approach to find the relationship between genetic variation and phenotype, has been used to open doors to the study of complex diseases and is also an important method of identifying genetic susceptibility genes in complex diseases. Professor Shen Hongbing of Nanjing Medical University has successively published many papers in NATURE GENETICS and other journals in which he elaborated the etiology and molecular epidemiology of chronic non-infectious diseases, especially those of tumors, through high-throughput gene sequencing and other techniques. Outstanding achievements have been made in the early diagnosis, genetic susceptibility and molecular markers of recurrence and prognosis of cancers, which is important for the pro- motion of tumor molecular diagnosis and tumor personalized therapy.

With the gradual deepening of the concept of precision medicine and the personalized medical model, the treatment of cancers is being gradually expanded from the traditional three major treatments to four major therapies which include surgery, chemotherapy, radiotherapy and immunotherapy. Tumor immunotherapy is a kind of anti-cancer therapy, which is designed to activate the body's immune system in order to kill cancer cells and tumor tissues by way of the selfimmune function. In 2013, cancer immunotherapy was recognized as number one in the top ten breakthroughs as appraised in SCIENCE. It is increasingly clear that the genetic problem of cancers is not wholly related to malignant tumors and this drives researchers to focus on cancer immunotherapy. Increasingly, exciting results have been made in antibody targeted therapy, tumor vaccines and CAR-T cell therapy and so on, bringing hope to the progressive advanced cancer patients.

Although milestone achievements to date in cancer research have brought hope to some cancer patients, we still lack knowledge for personalized treatment regimens for most tumors. Therefore, the discovery of more tumor target genes, the development of precision tumor molecular diagnosis and the establishment of personalized tumor medical model are the main directions of future tumor research.

(Received 08 October 2017, Revised 06 November 2017, Accepted 08 November 2017) 